\title{
Insights into the mechanism of a novel shockwave-assisted needle-free drug delivery device driven by in situ-generated oxyhydrogen mixture which provides efficient protection against mycobacterial infections
}

\author{
Janardhanraj Subburaj ${ }^{1+}$, Akshay Datey ${ }^{1,2,3+}$, Jagadeesh Gopalan ${ }^{1,3^{*}}$ and Dipshikha Chakravortty ${ }^{2,3^{*}}$
}

\begin{abstract}
Background: Needle-free, painless and localized drug delivery has been a coveted technology in the area of biomedical research. We present an innovative way of trans-dermal vaccine delivery using a miniature detonation-driven shock tube device. This device utilizes 2.5 bar of in situ generated oxyhydrogen mixture to produce a strong shockwave that accelerates liquid jets to velocities of about $94 \mathrm{~m} / \mathrm{s}$.

Method: Oxyhydrogen driven shock tube was optimized for efficiently delivering vaccines in the intradermal region in vivo. Efficiency of vaccination was evaluated by pathogen challenge and host immune response. Expression levels of molecular markers were checked by qRT-PCR.

Results: High efficiency vaccination was achieved using the device. Post pathogen challenge with Mycobacterium tuberculosis, 100\% survival was observed in vaccinated animals. Immune response to vaccination was significantly higher in the animals vaccinated using the device as compared to conventional route of vaccination.

Conclusion: A novel device was developed and optimized for intra dermal vaccine delivery in murine model. Conventional as well in-house developed vaccine strains were used to test the system. It was found that the vaccine delivery and immune response was at par with the conventional routes of vaccination. Thus, the device reported can be used for delivering live attenuated vaccines in the future.
\end{abstract}

Keywords: Shockwaves, Oxyhydrogen, Mycobacterium bovis BCG, Mycobacterium tuberculosis, Vaccination, Immune response

\section{Background}

The most commonly used needle and syringe method for drug administration has come under scrutiny in the recent decades because of factors like needle contamination, requirement for safe disposal of used needles, waste accumulation, accidental needle-stick, pain during

\footnotetext{
* Correspondence: jaggie@iisc.ac.in; dipa@iisc.ac.in

${ }^{\dagger}$ Equal contributors

'Department of Aerospace Engineering, Indian Institute of Science, Bangalore, India ${ }^{2}$ Department of Microbiology and Cell Biology, Indian Institute of Science, Bangalore, India

Full list of author information is available at the end of the article
}

usage and needle phobia [1]. Therefore, many new alternative non-invasive means of drug delivery have been developed, which mainly use oral, pulmonary, nasal, buccal or transdermal routes of administration [1-3]. Among these, the drug transport through human skin proves to be more advantageous compared to the other routes due to the ease of administration, immunosurveillance functions and easy accessibility $[2,4]$. Liquid-jet injectors, powder immunization and microneedles are some of the budding technologies for transdermal delivery of drugs [1-6]. Many methods to improve the efficiency of transdermal therapeutic systems by enhancing 
the driving force to increase the rate of drug transport have also been suggested [6, 7]. However, these techniques face major challenges due to the selectively permeable nature of the human skin and its ability to restrict molecular transport.

Shockwaves are non-linear waves, propagating at speeds greater than the speed of sound, with a unique characteristic of instantaneously increasing the pressure, temperature and density of the medium through which they propagate. For over a half a century, the phenomena of shockwaves have been synonymous with aerospace research $[8,9]$. The emerging paradigms of present day shock wave research have opened up new horizons for interdisciplinary applications [10]. Shockwaves have been extensively used for various medical procedures like extracorporeal lithotripsy [11], treatment of avascular necrosis [12], accelerated bone fracture healing [13], angiogenesis [14] and tendinitis [15]. The use of shockwaves as a driving force for transdermal drug delivery has proved to be effective because of their ability to accelerate the drug particles to high velocities so that they can penetrate the skin [16-21].Shockwaves have also been demonstrated to generate high velocity projectiles for delivering nucleic acids into living cells [22-25]. However, the use of either compressed air bottles [16-18], ignition of detonable mixtures [20-25] or operation of bulky and expensive instruments [19] to generate shockwaves, make these techniques undesirable. Also, many of the techniques result in the accumulation of waste and production of harmful by-products during the detonation of mixtures.

In the present work, we have developed a shockwaveassisted vaccine delivery device that can generate high velocity jets through the detonation of in situ generated oxyhydrogen mixture (stoichiometric mixture of hydrogen and oxygen gases in the ratio 2:1). BCG and Salmonella vaccine strains (DV-STM-07) [26] have been successfully injected using this device in the mice model. The device has been optimized to obtain penetration depths of $\sim 100 \mu \mathrm{m}$ in the skin. The molecular mechanism of the immune response to shockwave mediated vaccine delivery has been worked out. The ability of the proposed device to produce shockwaves of required strength in a safe, clean and reproducible manner opens up new opportunities for shockwave-assisted biomedical research. This device demonstrates the potential for localized drug delivery using shockwaves.

\section{Methods}

Operation of the oxyhydrogen-driven drug delivery device

The biological sample was placed in the sterile cavity and clamped at the end of the shock tube. Tracing paper was used between the driver and driven section of the shock tube. The electrolysis process in the oxyhydrogen generator was initiated when the power supply from the DC source was switched on. The oxyhydrogen generator produced stoichiometric ratio of hydrogen and oxygen gases at around $8 \mathrm{ml} / \mathrm{s}$. As soon as the fill pressure in the driver section of the shock tube reached2.5 bar, the power supply was switched off. By evacuating the pressure between the oxyhydrogen generator and the miniature shock tube, it was ensured that the detonation front does not travel into the oxyhydrogen generator when the shock tube is operated. The miniature shock tube was operated by igniting the mixture using a spark plug. For the subsequent run, the ruptured paper diaphragm was replaced with new ones. The biological sample was replaced with a new sample.

\section{Pressure measurements in the setup}

The pressure in the shock tube was measured using a piezoelectric pressure sensor (PCB Piezotronics, USA). This sensor was flushed mounted to the wall of the shock tube. To measure the head-on pressures experienced by the liquid in the cavity, the sensor was mounted on the base of the cavity. A polyvinyldifluoride (PVDF)-coated needle hydrophone (Dr. Müller Instruments, Germany) was used to measure the pressure experienced by the bacterial culture in the cavity. The needle gauge was placed in the cavity so that the tip of the sensor is in contact with the bacterial culture. All the pressure signals obtained from the sensors were recorded in an oscilloscope (Yokogawa Electric, Japan).

\section{High-speed visualization of the blast wave evolution and liquid jet}

A conventional Z-type Schlieren visualization technique that uses a high-speed camera (Phantom V310, Vision Research, USA) and an LED light source were used to obtain time resolved high speed images. The Schlieren system was triggered using the pressure sensor at the end of the driven section.

\section{Institutional ethical clearance and permission}

All the experiments were carried out in accordance with the institutional ethical guidelines. Ethical clearance for the project was obtained from the Institutional Animal Ethics Committee (IAEC; Reg. no. 48/1999/CPCSEA, Chairman: Prof. D N Rao). Permission no: CAF/Ethics/ $380 / 2014$.

\section{Effect of shock waves on the viability of bacteria}

Salmonella Typhimurium and Mycobacterium bovis BCG were the model microorganisms used to study the effect of shock waves on bacterial viability. Overnight cultures were pelleted and suspended in sterile phosphate buffered saline (PBS). The re-suspended culture was exposed to shockwaves at different pressures. The cultures were 
immediately aspirated, serially diluted and plated on SS (Salmonella-Shigella) agar or Middlebrook 7H10 agar. Equal volume of unexposed culture was used as a control. CFU were counted and was expressed as percent viability.

\section{Depth of penetration measurements}

To mimic the physical parameters corresponding to the animal skin, $20 \%$ Polyacrylamide gel was used to visualize the injection. Carboxylate-modified polystyrene fluorescent yellow-green Latex beads (L4655, SigmaAldrich) $\left(4.6 \times 10^{9}\right.$ particles per $\left.\mathrm{ml}\right)$ were injected into the polyacrylamide gel using the device. The gel was immediately visualized using a Carl Zeiss 710 confocal microscope to visualize and quantify the depth of penetration. Above mentioned latex beads were injected in vivo on the dorsal side of the animal. The skin was removed and mounted on a glass slide and immediately visualized using confocal microscope. Images were obtained in $x y z$ scanning mode and captured every $2 \mu \mathrm{m}$ from the skin surface until no appreciable fluorescence could be detected.

\section{Immunization of the mice}

$\mathrm{BALB} / \mathrm{c}$ mice were bred and housed at the Central Animal facility, Indian Institute of Science. The mice used for the experiments were 6-8 weeks old. All procedures with animals were carried out in accordance with the institution-approved rules. The abdominal region of the animal was shaved using hair removal cream. The skin was wiped with $70 \%$ ethanol before the vaccine delivery. DV-STM-07 and M. bovis BCG were used as vaccine strains. The mice were held in close proximity to the device. The device was operated at an optimum fill pressure of 2.5 bar to ensure the injection of the vaccine. Mice ( $n=5$ per group) were immunized using approximately $10^{3}$ bacteria/mouse. One group of mice was administered PBS which served as a control. Mice in the positive control group for DV-STM-07 vaccination received $10^{7} \mathrm{CFU} /$ mouse via the oral route. 1-week post immunization all the mice were challenged orally with $10^{7} \mathrm{CFU} / \mathrm{ml}$ and $10^{8} \mathrm{CFU} / \mathrm{ml}$ of virulent Salmonella Typhimurium for estimating the organ burden and for survival assay respectively. The mice were monitored twice daily for the morbidity and mortality.

\section{BCG vaccination}

BCG vaccine was administered to animals using the device (approximately $10^{3} \mathrm{CFU} /$ animal) as described above. A booster dose was given on day 7 post primary dose of the vaccine. Positive control group was administered the vaccine via intradermal route.

\section{Estimation of organ burden in BCG vaccination}

Day 14 post vaccination, animals were challenged with Mycobacterium tuberculosis $\mathrm{H} 37 \mathrm{Ra}\left(10^{7} \mathrm{CFU} /\right.$ mouse) via the nasal route using agarose bead slurry. Day 7 post infection, the animals were sacrificed using cervical dislocation method and the lungs were aseptically dissected, homogenized and plated on 7H10 agar. CFU were counted and were represented as $\mathrm{CFU} / \mathrm{g}$ organ weight.

\section{Estimation of organ burden in DV-STM-07 vaccination}

Five days' post oral challenge the mice were sacrificed by the cervical dislocation method ${ }^{32}$. The liver, spleen and mesenteric lymph nodes (MLN) were aseptically isolated, weighed and homogenized in sterile PBS. The homogenate was serially diluted and plated on SS agar to estimate the bacterial load in various organs. CFU were counted and represented as CFU/g wt. of the organ.

\section{Estimation of serum immunoglobulin-G (IgG)}

Blood was collected from the mice by retro-orbital ocular bleeding (33). Serum was isolated by centrifugation and stored at $-80{ }^{\circ} \mathrm{C}$ until use. The serum samples were diluted in PBS supplemented with $3 \%$ bovine serum albumin (BSA) prior to use in enzyme-linked immune sorbent assay (ELISA). Serum Immunoglobulin-G (IgG) titer specific to Salmonella lipopolysaccharide (LPS) and BCG was estimated using ELISA. LPS (250 ng/100 $\mu \mathrm{l} /$ well) in $0.02 \%$ trichloroacetic acid or BCG bacteria $\left(10^{3} \mathrm{CFU} / 100 \mu \mathrm{l} /\right.$ well $)$ were coated in a 96 well plate and incubated at $4{ }^{\circ} \mathrm{C}$ for $12 \mathrm{~h}$. The unbound sites on the plate were blocked with $3 \%$ BSA for $1 \mathrm{~h}$ at room temperature. $100 \mu \mathrm{l}$ of diluted serum $(1: 10)$ was added and incubated for $1 \mathrm{~h}$ at $37{ }^{\circ} \mathrm{C}$ and was washed. HRP conjugated anti IgG mouse (1:5000 dilutions) was added and incubated for $1 \mathrm{~h}$ at $37{ }^{\circ} \mathrm{C}$. The wells were washed with PBS with Tween 20 thrice. $100 \mu \mathrm{l}$ of tetramethylbenzidine solution was added and incubated for $15 \mathrm{~min}$. $50 \mu \mathrm{l}$ of $1 \mathrm{~N} \mathrm{H}_{2} \mathrm{SO}_{4}$ was added to stop the reaction and the absorbance was recorded at $540 \mathrm{~nm}$. The highest dilution at which the absorbance of the sample exceeds the background absorbance by 2 standard deviations was taken as end point titers of the sera.

\section{Estimation of $\mathrm{CD}^{+} \mathrm{T}$ cells}

Splenic $\mathrm{T}$ cells were isolated from unvaccinated and vaccinated mice as follows: Freshly harvested splenic tissue was physically disintegrated using roughened glass slides in tissue culture medium maintained at $4{ }^{\circ} \mathrm{C}$. Single cell suspension was made by passing the cell suspension though a filter. RBC lysis was done using RBC lysis buffer. After lysis, cells were allowed to adhere to culture dishes for $1 \mathrm{~h}$. Supernatant containing T cells was collected. Cells were briefly washed with sterile PBS and 
were fixed using 3\% paraformaldehyde for $15 \mathrm{~min}$ and were stored at $4{ }^{\circ} \mathrm{C}$ until further use. Cells were stained with PE tagged antiCD8 antibody (MiltenyiBiotec) and were analyzed by flow cytometry. Numbers of CD8+ cells were compared between the unvaccinated and the vaccinated cohort.

\section{Quantitative real time PCR}

Tissue was aseptically isolated from the animal and was immediately resuspended in Trizol (Invitrogen) and stored in $-80{ }^{\circ} \mathrm{C}$ till further use. RNA was isolated and converted to cDNA using reverse transcriptase. Real time PCR was set up using SyBr green kit from Thermo Scientific and was run on Applied Biosystems VIIA. The fold expression was normalized to $ß$-actin.

\section{ELISA for serum cytokines}

Blood was collected through the retro-orbital route. Serum was isolated by centrifugation and was stored at $-80{ }^{\circ} \mathrm{C}$ until use. BD Opt ELISA kit was used for determining the levels of cytokines in the serum.

\section{Statistical analysis}

The data sets were statistically analyzed by applying Student's t-test and Mann-Whitney U test using Graph Pad prism 5 software. A $p$-value $<0.05$ was considered as significant. The mortality and survival data were analyzed by survival curve analysis. All the experiments were repeated at least thrice to validate the results.

\section{Results}

Design of the oxyhydrogen detonation-driven drug delivery device

The oxyhydrogen detonation-driven drug delivery device comprises of two main components namely oxyhydrogen generator and miniature shock tube assembly. While the design and working of the oxyhydrogen generator used for the present work has already been described [27], some changes have been made in the miniature shock tube assembly to facilitate vaccine delivery experiments (Fig. 1). Through alkaline electrolysis, the oxyhydrogen generator produces about 2.5 bar of oxyhydrogen mixture during each operation of the device. The miniature shock tube of internal diameter $6 \mathrm{~mm}$ comprises of two sections - a driver section of length $200 \mathrm{~mm}$ and driven section of length $70 \mathrm{~mm}$ (Fig. 1). Tracing paper ( 95 GSM) is used as diaphragm to separate the driver section and driven section of the shock tube. To facilitate quicker and easier changing of diaphragm after each experiment as well as filling the drug in the cavity, a tri-clover clamp is used between the different sections of the shock tube. The driver section of the shock tube is filled with 2.5 bar of oxyhydrogen mixture and the mixture is detonated. This ruptures the diaphragm and a strong shockwave is created in the driven section of the shock tube. The working of the miniature shock tube is explained in a schematic diagram (see Additional file 1: Figure S1). The vaccine is accommodated in a stainless steel sterile cavity of diameter $6 \mathrm{~mm}$ and depth $5 \mathrm{~mm}$ (Fig. 1). The dimensions of the cavity is fixed based on a study performed earlier [21]. The bottom of the cavity has a $300 \mu \mathrm{m}$ diameter hole to allow vaccine to be ejected in the form of a jet on shockwave impact. The drug is held back initially in the cavity by surface tension. The direct impact of the shockwave followed by the products of detonation on the drug sample leads to issues of contamination. To avoid this, a suitable barrier is chosen for good energy transfer and to prevent the detonation products from impacting the drug. Using a brass foil is a viable option [20] but requires replacement after every experiment and also it absorbs most of the incident shockwave energy before transmitting it to the biological sample. Therefore, in the present workman silicone rubber membrane has been used between the shock tube and the drug. Silicone rubber has biocompatible properties and very good tensile strength [28].These properties make it ideal for the present work as it need not be replaced frequently and the energy transfer is better as compared to using the brass foil.

\section{Performance of the oxyhydrogen detonation-driven drug delivery device}

The initial fill pressure of the oxyhydrogen mixture in the shock tube determines the strength of the shockwave produced which, in turn, determines the amount of energy transferred to the vaccine. In order to fix the initial fill pressure of the oxyhydrogen mixture for the experiments in the present study, we delved into the working of a previously reported shockwave-assisted drug delivery device that uses a Nonel tube to generate shockwaves. After an elapsed time of $50 \mu \mathrm{s}$, the blast wave emanating from the open end of the Nonel tube carries an energy of about $1.25 \pm 0.94 \mathrm{~J}$ [29]. The energy of the blast waves produced using the present device was targeted to be around this value. The initial experiments using the miniature shock tube assembly was carried without the cavity, silicone rubber and the vaccine. The driven section was left open to the atmosphere to analyze the blast waves coming out of the open end of the shock tube. The miniature shock tube was operated with an initial oxyhydrogen fill pressure of 2.5 bar. Shockwaves with a peak pressure of $14.68 \pm 1.28$ bar were produced with good reproducibility at the end of the shock tube (Fig. 2a). The energy of the shockwave was estimated from the pressure-plot of the shockwave based on a procedure that has been reported elsewhere [30]. The energy of the blast waves emanating from the 


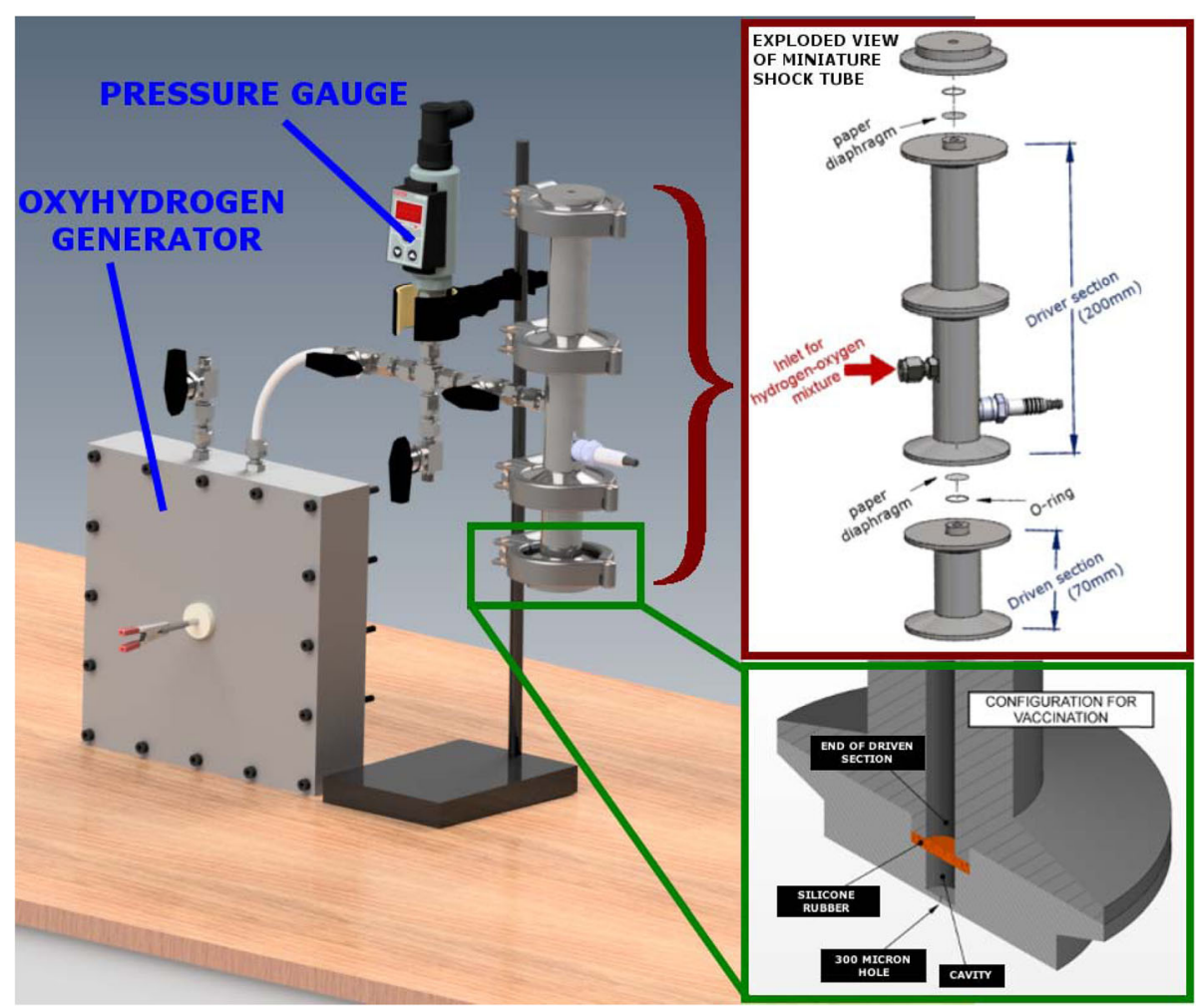

Fig. 1 Schematic of the oxyhydrogen detonation-driven drug delivery device. Schematic of the entire experimental setup showing the various subcomponents. Cross-sectional view of the end of the miniature shock tube assembly showing the flexible silicone rubber and the 300- $\mu \mathrm{m}$ hole in the cavity

open end was estimated to be $18.86 \pm 1.35 \mathrm{~J}$ after $500 \mu \mathrm{s}$ (Fig. 2b). After $50 \mu \mathrm{s}$, the energy in the blast wave was about $4 \mathrm{~J}$ which is almost three times the targeted value. High speed schlieren photography [31] of the open end of the shock tube revealed the symmetrical nature of the blast wave about the axis of the shock tube(Fig. 2c and Additional file 2: video 1). The stronger color gradients indicate the higher strength of the primary and secondary blast waves emanating as a result of the oxyhydrogen detonation. Also, the cloud of detonation products behind the secondary blast waves could be observed in the photographs.

\section{Characterization of liquid jet delivery in artificial and animal skin}

High-speed images of the liquid jet were acquired using conventional Schlieren technique for oxyhydrogen initial fill pressure of 2.5 bar (Fig. $2 \mathrm{~d}$ and Additional file 3: video 2 ). The velocity calculation from the images gave a value of $94 \pm 9 \mathrm{~ms}^{-1}$ (Fig. 2e). The targeted values for velocity of jet and depth of penetration are $\sim 100 \mathrm{~ms}$ ${ }^{-1}$ and $100-150 \mu \mathrm{m}$ respectively [20]. The aim of needleless vaccination is to deliver the biological sample to the stratum corneum layer of the skin which is known to be devoid of nerve endings responsible for the sensation of pain [5]. This portion of the skin is populated by special immune cells called Langerhans cells. These are the "professional" antigen presenting cells operating in the dermal region [5]. To check the depth of penetration of the liquid jet, initially 20\% Polyacrylamide gel target (a known artificial skin model [32, 33]) was used. This gel has similar mechanical properties as compare to the human skin. The Young's modulus of the human skin ranges from 0.02 to $57 \mathrm{MPa}$ and that of the polyacrylamide gel used is $<60 \mathrm{MPa}$ [32]. Green fluorescent beads ( $1 \mu \mathrm{m}$ diameter) were injected into the gel using the shock tube. The depth of penetration was evaluated using confocal microscopy, which was found out to be approximately $120 \mu \mathrm{m}$ (Fig. 3a). This depth in the skin is the most suitable for painless injections. To validate the same in vivo, the beads were injected in the mice, the skin was dissected immediately and was analyzed using confocal microscope. The maximum depth of penetration of the beads was found out to be $80 \mu \mathrm{m}$ which was reasonably sufficient to deliver the vaccine to the resident Langerhans's cells for generating an immune response (Fig. 3b).

\section{Efficiency of BCG vaccination delivery using the device} $B C G$ vaccine is conventionally administered in the intradermal region. Using shock waves to deliver this vaccine 


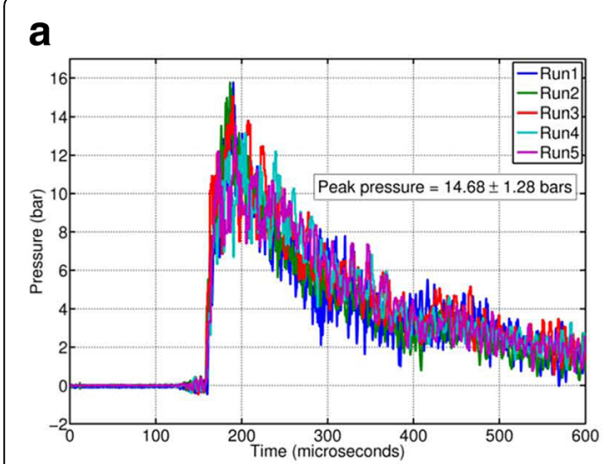

b

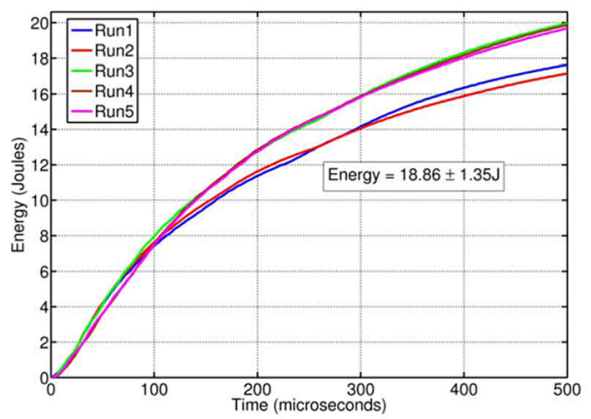

d

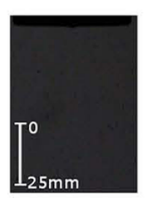

$0 \mathrm{~ms}$

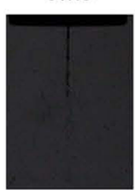

$1.76 \mathrm{~ms}$

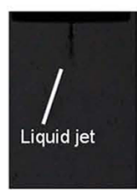

$0.58 \mathrm{~ms}$

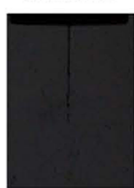

$2.35 \mathrm{~ms}$

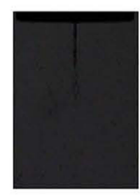

$1.17 \mathrm{~ms}$

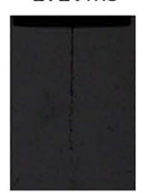

$2.94 \mathrm{~ms}$ e

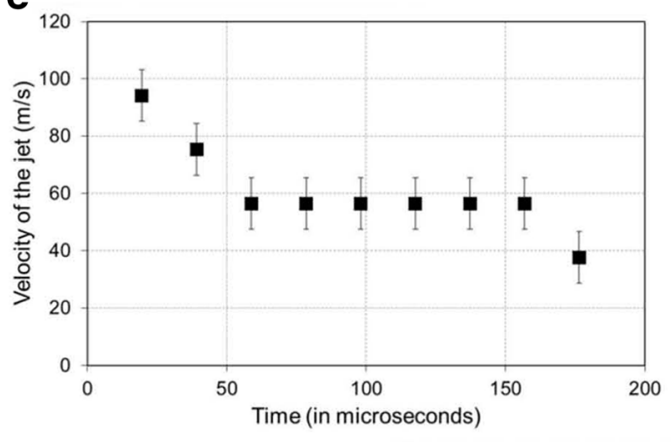

Fig. 2 Measurements made in the oxyhydrogen detonation-driven miniature shock tube. a Typical pressure signals for five different runs of the shock tube operated at an oxyhydrogen fill pressure of 2.5 bar. The pressure sensor is flush-mounted to the inside wall of the driven section at a distance of $12 \mathrm{~mm}$ from the end. The driven section is left open to the atmosphere. $\mathbf{b}$ The corresponding energies in the shock wave for the five runs. c Time resolved high speed Schlieren images of the blast wave emanating from the open end of the shock tube. The images are acquired at a frame rate of $40,000 \mathrm{fps}$. d Time-resolved high speed shadowgraph images of the jet of drug ejected through the 300- $\mu \mathrm{m}$ hole. The images are acquired at a frame rate of 51,000 fps. e A velocity-time plot of the tip of the jet as measured from the high-speed shadowgraphy

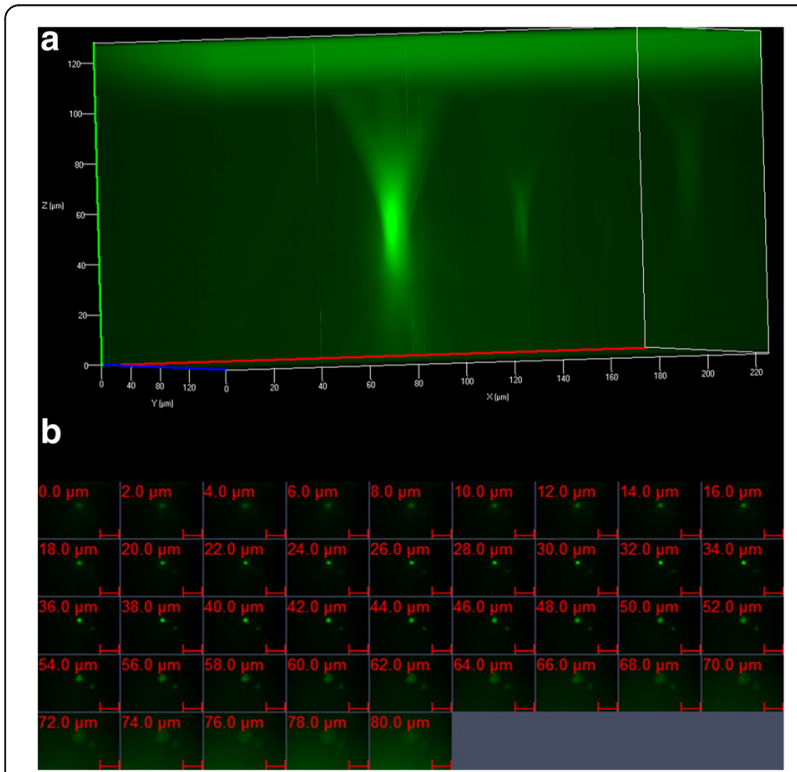

Fig. 3 a Confocal images showing the penetration of fluorescent yellow-green latex beads delivered to $20 \%$ acrylamide gels using the device. $\mathbf{b}$ Confocal images (xyz scanning) showing the penetration of fluorescent beads delivered to the abdominal region of mouse skin using the device was more clinically relevant. We began with checking the effect of shockwaves on the viability of the vaccine strain. It was observed that shock waves had no effect on the viability of the vaccine strain (Fig. 4a). Animals were vaccinated using the device in the intradermal region of the abdomen. A booster dose was also administered to enhance the immune protection. The antibody titer against BCG was found significantly higher in the cohort vaccinated using the device as compared to the conventionally vaccinated route (Fig. 4b). This highlights the enhanced immune protection conferred when vaccinated using the device. To validate the immune response further, the vaccinated animals were challenged with $M$. tuberculosis $H 37 R a$ via the nasal route. The bacterial burden in the lungs of the animals vaccinated using the device was significantly lower than that of the conventionally vaccinated group as well as the control group (Fig. 4c). The CD8+ cells were estimated in from the spleen. There was a significant increase in the cytotoxic $\mathrm{T}$ cells in the vaccinated cohort in comparison to the control group (Fig. 4d-f). To perform a stringent test of vaccination, mice vaccinated with BCG using the device as well as the conventional intradermal route were infected via the nasal route with a lethal dose of virulent 


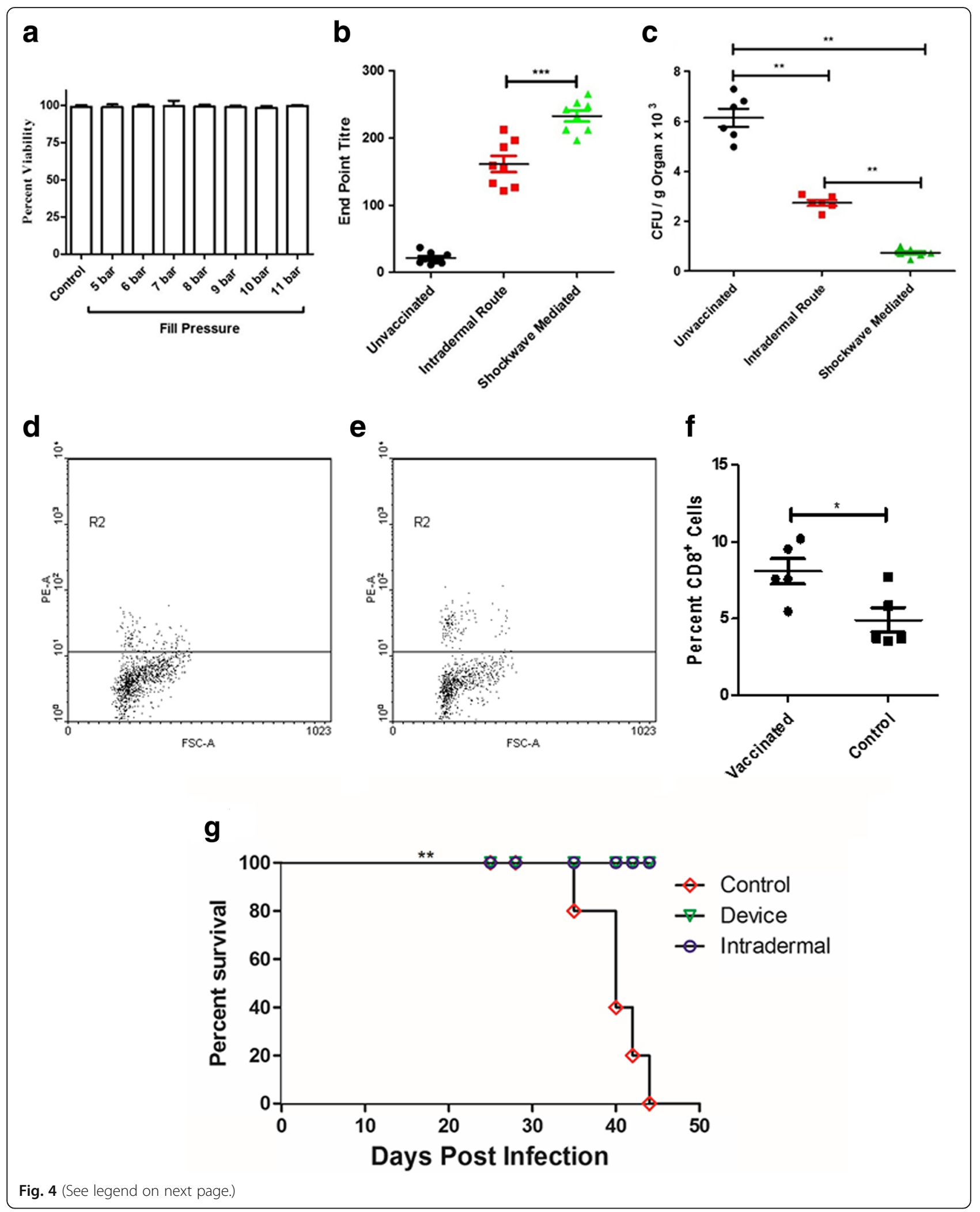




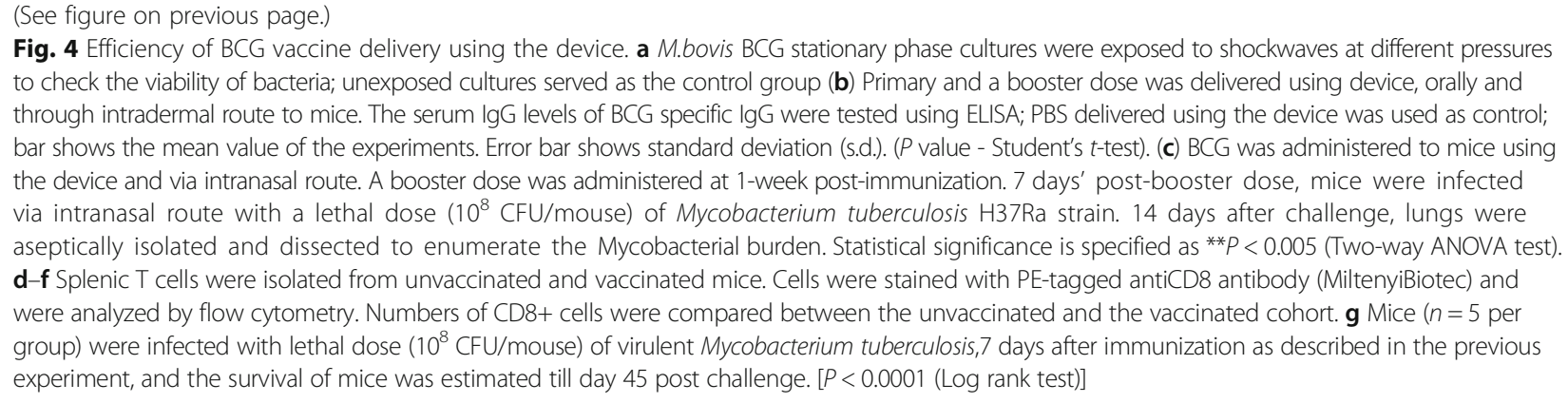

Mycobacterium tuberculosis $\mathrm{H} 37 \mathrm{Rv}$ and were monitored for mortality. A group of animals were injected PBS as a mock vaccination. It was observed that the animals vaccinated using the device and the ones vaccinated via the intradermal route showed $100 \%$ survival as compared to the mock vaccinated group. The entire control group succumbed to infection by day 44 post challenge (Fig. 4g). All these results prove that vaccination done using shockwave device is highly efficient in all the model organisms and infections tested.

\section{Efficiency of salmonella vaccine delivery using shockwave device}

Shockwaves had no significant effect on the viability of the vaccine strain DV-STM-07 (Fig. 5a). The survival rate of mice immunized using the device was similar to the group immunized via the oral and the IP route, whereas the mice from the control group did not survive when challenged with virulent Salmonella (Fig. 5b). This clearly proves the improved efficiency of the vaccination performed using the device. The bacterial colonization in liver, spleen and mesenteric lymph nodes of mice vaccinated using the device was significantly lower than that in the control, as well as, in the mice immunized by the oral and IP route (Fig. 5c). The level of serum IgG in the mice vaccinated using the shockwave devices were significantly higher than in the other modes of vaccination (Fig. 5d). The number of CD8+ cells in the cohort of animals vaccinated using the device was significantly high as compared to the animals vaccinated via the conventional oral route (Fig. $5 \mathrm{e}-\mathrm{g}$ ).

\section{Molecular mechanism of enhanced immune response to vaccine administered using shockwave device}

The immune response generated by the shockwave mediated vaccine delivery was found to be significantly higher than the conventional intradermal injections of the same vaccine. This intrigued us to further study the effect of shockwave in priming the immune system. In order to study the effect mentioned above, we designed the experiment where a cohort of animals was administered PBS using the shockwave device. A similar cohort was administered PBS using a hypodermal needle syringe. Post vaccination cytokine levels were checked in the animals. Cytokines namely, IL- 8 , IFN- $\gamma$, TNF- $\alpha$ and IL-2 were compared. These cytokines are important in generating a potent immune response by activating the immune cells. The levels of these cytokines were found to be high in the vaccine delivered using the device as compared to vaccine delivered using hypodermic syringe (Fig. 6a-d). Comparison of the above two cohorts was also done at the molecular level where the expression of genes like CD207, CD14, TGF- 3 , MHC-II from the site of injection $24 \mathrm{~h}$ post injection (Fig. 6e) and CD4, CD8. CD19, IFN $\gamma$ and MHC-II were evaluated from the superficial inguinal lymph nodes day 3 post injection using qRT-PCR (Fig. 6g). These factors mainly reflect the status of immune response generated against an antigen introduced through the dermal route. CD207 is the exclusive marker for skin resident dendritic cells called as the Langerhans's cells [34]. These cells are the most potent antigen presentation machinery present in the skin. MHC-II is the major histocompatibility complex associated with exogenous antigen processing and presentation [34]. CD4 and CD8 are the markers for helper T cells and cytotoxic T cells respectively. CD19 is the surface marker for antibody producing plasma cells [35]. It was observed that the markers tested at the site of injection were up- regulated at $24 \mathrm{~h}$ post injection in the PBS injection using device as compared to PBS injection using hypodermic syringe (Fig. 6f). Cohort injected with the vaccine strain showed significant upregulation of the markers. Day 4 post injection, molecular markers in the lymph nodes was quantitated. CD4, CD8, MHC-II, CD19 and IFN- $\gamma$ all were found upregulated in the PBS injection using device cohort. The up-regulation was significant in the cohort injected with vaccine strain using the device (Fig. 6h). This upregulation of the immune markers even in the case of PBS injection using the device clearly demonstrates that the shockwave and the high velocity jet which hits the site of injection activate the immune system. This might be because of the fact that shockwaves are recognised as a condition of physical stress and various stress responses 


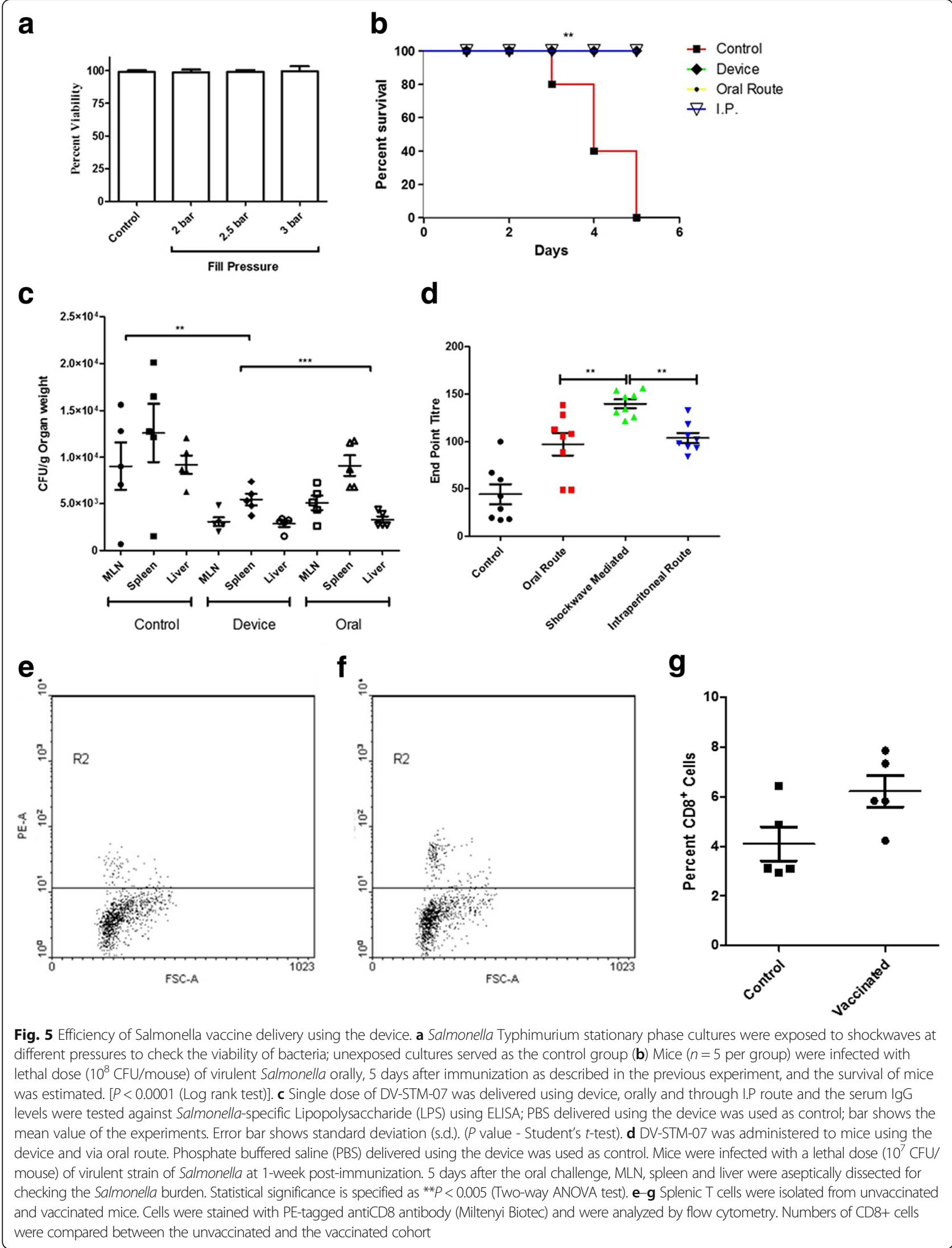




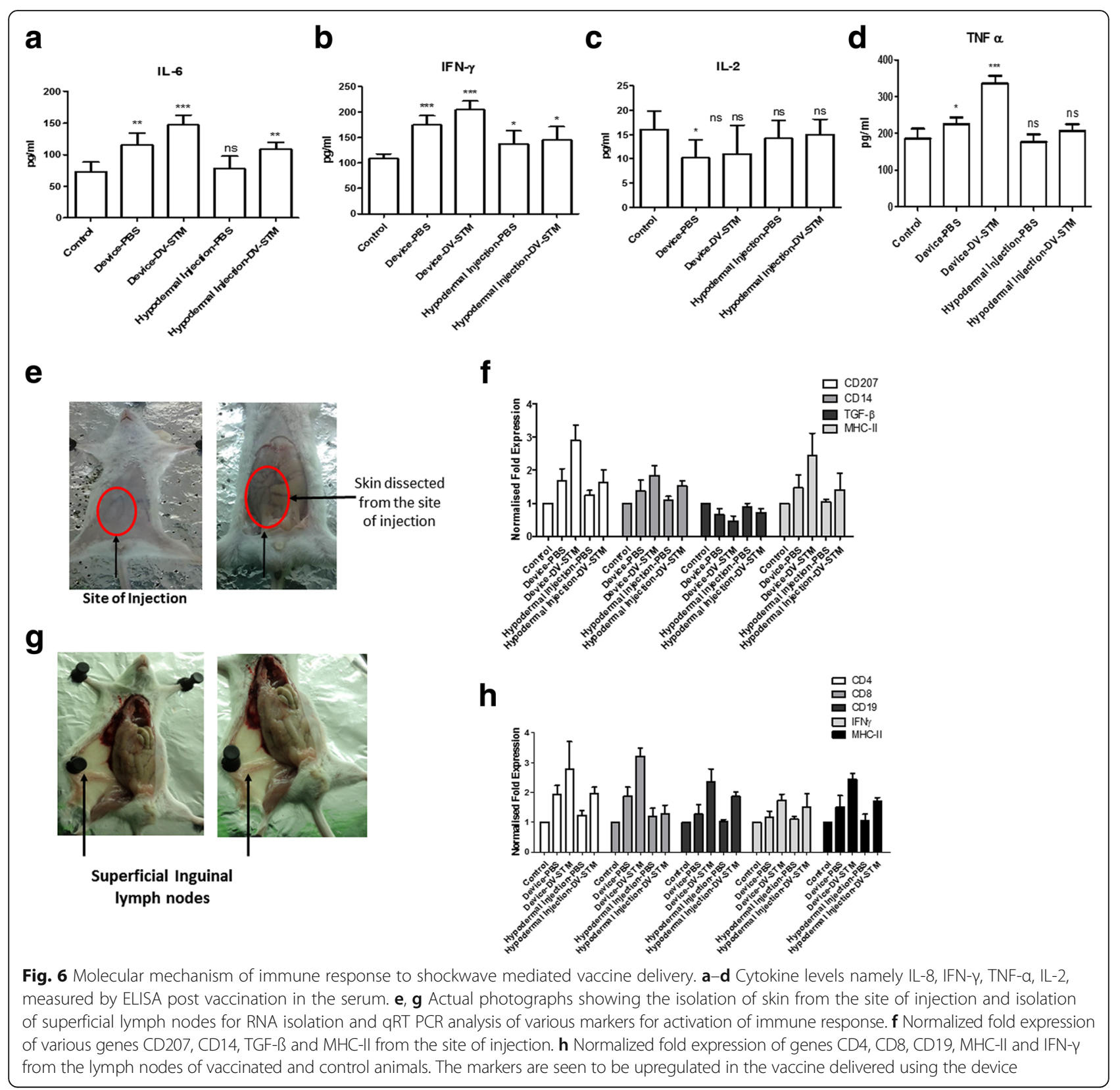

are rapidly mounted against shockwaves used for vaccine delivery. Therefore, all these results highlight the role of shockwaves in eliciting an immune response independent of the vaccine being administered.

\section{Mechanism of enhanced vaccine delivery using the present device}

The shockwave produced by the detonation of the oxyhydrogen mixture is the driving force for needleless vaccination. A large portion of the incident shockwave energy passes through the silicone rubber and is felt by the vaccine placed in the cavity. While some part of the incident energy is absorbed by the membrane itself another portion of the energy is reflected back into the shock tube. Additional file 1: Figure S1 shows the pressure experienced by the vaccine placed in the cavity during operation of the device. The incident shockwave in the driven section of the shock tube has a peak pressure about 16 bar (See Fig. 2a). But it gets amplified to about 200 bar in the liquid as seen in Additional file 1: Figure S2. The pulse duration lasts for about 40 microseconds. The rise in pressure inside the cavity to a pressure of up to 200 bar could be due to the combined effect of the transmitted shockwave and the pressure rise in the liquid due to the bulging of the rubber membrane. The gradual pressure drop after the peak value is due to rubber going back to 
its original shape and thus creating a pressure relieving effect in the cavity. There are some high frequency oscillations can be observed in the fall time duration which slowly dampen out with time. This could be because of the vibration of the silicone membrane or the multiple reflections in the cavity. The time period of these oscillations corresponds to the multiple reflections of pressure waves in the cavity rather than the natural frequency of vibration of silicone rubber (see Additional file 1: Note S1 and Note S2). Therefore, the transmitted shock wave undergoes a series of reflections against the bottom of the cavity and the face of the rubber membrane causing high frequency oscillations in the pressure inside the cavity and also continuously attenuating in intensity. Keeping all these points in mind, illustrations of the possible mechanism of vaccination using the shockwave assisted device have been shown in Additional file 1: Figure S3.

\section{Discussion}

Shockwave-assisted vaccine delivery surpasses the present-day needle and syringe technique of injections as it is needle-free, painless, eliminates the need for non-biodegradable waste disposal, avoiding the risk of disease transmission and there is no damage to cell tissues around the region of injection. In addition to these advantages, the proposed device uses in situ generation of oxyhydrogen mixture which avoids storage and mixing of detonable gases. The device uses minimal consumables, produces water as a main by-product during usage and is safe as well as reproducible. All these advantages coupled with the innovative design make it a unique and first-of-its-kind device. The device was optimized to deliver vaccines in the transdermal region which lies at a depth of $\sim 100 \mu \mathrm{m}$ from the skin surface. Characterization of the shockwave generated was done using the conventional Schlieren imaging technique. The velocity of the fluid jet formed, was measured using a high-speed camera and was found out to be $94 \mathrm{~ms}^{-1}$. Theoretically calculated values of velocity of the liquid jet gives similar results (see Additional file 1: Note S3 and Figure S2). The depth of deposition of the fluid using the shockwave device was measured in both artificial skin model of polyacrylamide and in the actual murine skin by injecting fluorescently-labeled beads. The depths of fluid delivery were found out to be $120 \mu \mathrm{m}$ and $80 \mu \mathrm{m}$ in the artificial skin and murine skin respectively. These depths are also indicative of the absence of free nerve endings thus making the delivery system pain-free. The depth of penetration of the liquid is directly proportional to the kinetic energy of the fluid jet which in turn is governed by the strength of the shockwave driving the formation of the jet. The strength of the shockwave can be tuned so as to achieve the desired depth of penetration of the liquid jet. Further, vaccine delivery was tested in the murine model for two well established bacterial vaccines namely, DV-STM-07 and the BCG vaccine. Suitable animal models were chosen for the experiments. Vaccinations performed by using the device were scored at all possible levels of a virulent challenge by Salmonella Typhimurium and Mycobacterium tuberculosis H37Ra. The survival of the vaccinated animals was at par with the cohort vaccinated by the conventional methods. Immune response is measured by the production of pathogen-specific immunoglobulins in the host. High level of antibody production indicates the robustness of vaccination. The IgG level in the shockwave mediated vaccine delivery was found out to be significantly higher as compared to the vaccination by the conventional route in both DV-STM-07 and BCG vaccine strains. Next, the efficiency of the vaccination lowering the pathogen burden in the systemic organs was evaluated. It was found that the animals vaccinated using the device had lower level of pathogen colonization in liver, spleen and mesenteric lymph nodes for Salmonella and in the lungs for M.tuberculosis H37Ra which wasstatistically significant as well. Production of cytotoxic $\mathrm{T}$ cells $\left(\mathrm{CD} 8^{+}\right.$cells) also indicates an elicited immune response. These cells in the splenic cell population were enumerated in the vaccinated and the control cohort of animals. The vaccinated cohort showed a statistically significant higher number of these cells as compared to the control group for both the vaccine strains. The vaccination efficiency was also evaluated by challenging the vaccinated animals with virulent Mycobacterium tuberculosis. It was observed that the vaccinated animals show a $100 \%$ survival as compared to the mock vaccinated cohort. Insights into the molecular mechanism of immune response to vaccine delivered using the device revealed the role of shockwaves alone in priming the immune response. Cytokine profile in the animals vaccinated using shockwave device show a significant increase as compared to control and hypodermal syringe injections. The molecular markers like CD207, CD19, CD4, CD8 etc. indicative of the status of immune system showed significant upregulation in the animals vaccinated using the shockwave device. These observations form firm evidence that the shockwave device developed in this study bears the potential to deliver a range of vaccines and drugs to the desired locations by altering the operating conditions. This device has a promising future in assisting clinicians and healthcare professionals to perform large scale needle-less and pain-free vaccine/drug delivery. The recent advances in photo electrolysis of water and development of new photoelectrode materials can aid in further miniaturization and increased efficiency of the device. 


\section{Conclusion}

A novel device was developed and optimized for intra dermal vaccine delivery in murine model. Conventional as well in-house developed vaccine strains were used to test the system. It was found that the vaccine delivery and immune response was at par with the conventional routes of vaccination. Thus, the device reported can be used for delivering live attenuated vaccines in the future.

\section{Additional file}

Additional file 1: Supplementary Dataset. Figure S1. Working principle of the oxyhydrogen detonation-driven miniature shock tube. Figure S2. A plot showing the velocity of the jet calculated theoretically from the pressure measured

inside the cavity. Figure S3. Proposed mechanism enhanced vaccine delivery using the device. NOTE S1. Estimation of natural frequency of silicone rubber clamped at the edges. NOTE S2. Estimation of time taken by stress waves to travel along liquid column. NOTE S3. Theoretical estimation of velocity of liquid jet. (DOCX $438 \mathrm{~kb}$ )

Additional file 2: video 1. Schlieren video of the blast evolution from the open end of the device. The initial oxyhydrogen fill pressure is 2.5 bars. (XSPF 564 bytes)

Additional file 3: video 2. Schlieren video of the liquid jet containing the drug. The initial oxyhydrogen fill pressure of 2.5 bars. (XSPF 564 bytes)

\section{Abbreviations}

BCG: Bacillus Calmette-Guérin; CD: Cluster of differentiation; CFU: Colony forming unit; IgG: Immunoglobulin G; IP: Intraperitoneal; LPS: Lipopolysaccharides; PBS: Phosphate buffered saline; PCR: Polymerase chain reaction; qRTPCR: Quantitative real time polymerase chain reaction; RBC: Red blood cell

\section{Acknowledgements}

The authors would like to acknowledge the research grants from Defense Research and Development Organization (DRDO) to JG, Life Science Research Board (LSRB0008) and DBT-IISC partnership program for advanced research in biological sciences and bioengineering to DC. Infrastructure support from ICMR (Center for Advanced Study in Molecular Medicine), DST (FIST), and UGC (specia assistance) is acknowledged. AD is supported by a fellowship from Department of Biotechnology, India. DC received DAE SRC outstanding Investigator award and funds.

\section{Availability of data and materials}

Please contact author for data requests.

\section{Authors' contributions}

A.D. and J.S. contributed equally to this work. D.C. and J.G. conceived the study and designed the experiments. J.S. and A.D. performed the experiments. A.D. performed the biology experiments and analysis. All authors contributed to analyzing the data and writing the manuscript. All authors read and approved the final manuscript.

\section{Ethics approval}

An ethics approval was taken for conducting animal experiments.

\section{Consent for publication}

This publication does not contain any individual person's data.

\section{Competing interests}

The authors declare that they have no competing interests.

\section{Publisher's Note}

Springer Nature remains neutral with regard to jurisdictional claims in published maps and institutional affiliations.

\section{Author details}

'Department of Aerospace Engineering, Indian Institute of Science, Bangalore, India. 2Department of Microbiology and Cell Biology, Indian Institute of Science, Bangalore, India. ${ }^{3}$ Centre for Biosystems Science and Engineering, Indian Institute of Science, Bangalore, India.

Received: 3 October 2017 Accepted: 15 November 2017

Published online: 12 December 2017

\section{References}

1. Mitragotri S. Immunization without needles. Nat Rev Immunol. 2005;5:905-16.

2. Zelikin AN, Ehrhardt C, Healy AM. Materials and methods for delivery of biological drugs. Nat Chem. 2016:8:997-1007.

3. Mitragotri S, Burke PA, Langer R. Overcoming the challenges in administering biopharmaceuticals: formulation and delivery strategies. Nat Rev Drug Discov. 2014;13:655-72.

4. Arora A, Prausnitz MR, Mitragotri S. Micro-scale devices for transdermal drug delivery. Int J Pharm. 2008:364:227-36.

5. Mitragotri S. Current status and future prospects of needle-free liquid jet injectors. Nat Rev Drug Discov. 2006;5:543-8.

6. Barry BW. Novel mechanisms and devices to enable successful transdermal drug delivery. Eur J Pharm Sci. 2001;14:101-14.

7. Gratieri T, Alberti I, Lapteva M, Kalia YN. Next generation intra- and transdermal therapeutic systems: using non- and minimally-invasive technologies to increase drug delivery into and across the skin. Eur J Pharm Sci. 2013; 50:609-22.

8. Bradley JN. Shock waves in chemistry and physics. Methuen: Wiley; 1962.

9. Gaydon AG, Hurle IR. The shock tube in high-temperature chemical physics. Springer: New York; 1963.

10. Jagadeesh G. Fascinating world of shock waves. Resonance. 2008;13:752-67.

11. Thomas K. Evolution of extracorporeal shockwave lithotripsy (ESWL). BJU Int. 2014;114:636.

12. Wang CJ, Ko JY, Chan YS, Lee MS, Chen JM, Wang FS, Yang KD, Huang CC. Extracorporeal shockwave for hip necrosis in systemic lupus erythematosus. Lupus. 2009:18:1082-6.

13. Chow DH, Suen PK, Fu LH, Cheung WH, Leung KS, Wong MW, Qin L. Extracorporeal shockwave therapy for treatment of delayed tendon-bone insertion healing in a rabbit model: a dose-response study. Am J Sports Med. 2012:40:2862-71

14. Wang CJ, Huang KE, Sun YC, Yang YJ, Ko JY, Weng LH, Wang FS. VEGF modulates angiogenesis and osteogenesis in shockwave-promoted fracture healing in rabbits. J Surg Res. 2011;171:114-9.

15. Farr S, Sevelda F, Mader P, Graf A, Petje G, Sabeti-Aschraf M. Extracorporeal shockwave therapy in calcifying tendinitis of the shoulder. Knee Surg Sports Traumatol Arthrosc. 2011;19:2085-9.

16. Quinlan N, Kendall M, Bellhouse B, Ainsworth R. Investigations of gas and particle dynamics in first generation needle-free drug delivery devices. Shock Waves. 2001;10:395-404

17. Kendall MA. The delivery of particulate vaccines and drugs to human skin with a practical, hand-held shock tube-based system. Shock Waves. 2002:12:23-30.

18. Kendall M, Mitchell T, Wrighton-Smith P. Intradermal ballistic delivery of micro-particles into excised human skin for pharmaceutical applications. J Biomech. 2004;37:1733-41.

19. Menezes V, Takayama K, Ohki T, Gopalan J. Laser-ablation-assisted microparticle acceleration for drug delivery. Appl Phys Lett. 2005;87:163504.

20. Jagadeesh G, Prakash GD, Rakesh SG, Allam US, Krishna MG, Eswarappa SM, Chakravortty D. Needleless vaccine delivery using micro-shock waves. Clin Vaccine Immunol. 2011;18:539-45.

21. Rakesh SG, Gnanadhas DP, Allam US, Nataraja KN, Barhai PK, Jagadeesh G, Chakravortty D. Development of micro-shock wave assisted dry particle and fluid jet delivery system. Appl Microbiol Biotechnol. 2012;96:647-62

22. Klein TM, Wolf E, Wu R, Sanford J. High-velocity microprojectiles for delivering nucleic acids into living cells. Nature. 1987;327:70-3.

23. Boynton JE, Gillham NW, Harris EH, Hosler JP, Johnson AM. Chloroplast transformation in Chlamydomonas with high velocity microprojectiles. Science 1988:240:1534.

24. Klein TM, Fromm M, Weissinger A, Tomes D, Schaaf S, Sletten M, Sanford JC Transfer of foreign genes into intact maize cells with high-velocity microprojectiles. Proc Natl Acad Sci. 1988;85:4305-9. 
25. Mendel R, Müller B, Schulze J, Kolesnikov V, Zelenin A. Delivery of foreign genes to intact barley cells by high-velocity microprojectiles. Theor Appl Genet. 1989;78:31-4.

26. Negi VD, Nagarajan AG, Chakravortty D. A safe vaccine (DV-STM-07) against salmonella infection prevents abortion and confers protective immunity to the pregnant and new born mice. PLoS One. 2010;5:e9139.

27. Janardhanraj S, Jagadeesh G. Development of a novel miniature detonationdriven shock tube assembly that uses in situ generated oxyhydrogen mixture. Rev Sci Instrum. 2016;87:085114.

28. Mohanan PV, Rathinam K. Biocompatibility studies on silicone rubber. Proceeding of the First Regional Conference - leee Engineering in Medicine \& Biology Society and. In: 14th conference of the Biomedical Engineering Society of India; 1995. p. 369-70.

29. Samuelraj IO, Jagadeesh G, Kontis K. Micro-blast waves using detonation transmission tubing. Shock Waves. 2013:23:307-16

30. Wang E, Shukla A. Analytical and experimental evaluation of energies during shock wave loading. International Journal of Impact Engineering. 2010;37:1188-96.

31. Settles GS. Schlieren and shadowgraph techniques: visualizing phenomena in transparent media: Springer Science \& Business Media: New York; 2012. http://www.springer.com/gp/book/9783540661559.

32. Schramm-Baxter J, Katrencik J, Mitragotri S. Jet injection into polyacrylamide gels: investigation of jet injection mechanics. J Biomech. 2004;37:1181-8.

33. Baxter J, Mitragotri S. Jet-induced skin puncture and its impact on needle-free jet injections: experimental studies and a predictive model. J Control Release. 2005;106:361-73.

34. Liard C, Munier S, Joulin-Giet A, Bonduelle O, Hadam S, Duffy D, Vogt A, Verrier B, Combadiere B. Intradermal immunization triggers epidermal Langerhans cell mobilization required for CD8 T-cell immune responses. J Invest Dermatol. 2012;132:615-25.

35. Itzchak S, Jacob B, Avraham R, Ben-Ami P. Enhancement of the immune response by intradermal vaccination in cattle with enterotoxigenic Escherichia Coli (ETEC) vaccine without adjuvant. Vaccine. 1992;10:217-20.

\section{Submit your next manuscript to BioMed Central and we will help you at every step:}

- We accept pre-submission inquiries

- Our selector tool helps you to find the most relevant journal

- We provide round the clock customer support

- Convenient online submission

- Thorough peer review

- Inclusion in PubMed and all major indexing services

- Maximum visibility for your research

Submit your manuscript at www.biomedcentral.com/submit

) Biomed Central 